\title{
An investigation of the effects of walking frame height and width on walking stability.
}

Sibylle Brunhilde Thies ${ }^{a *}$, Rachel Russel ${ }^{a}$, Abdullah Al-Ani ${ }^{b}$, Tom Belet $^{c}$, Alex Bates ${ }^{a}$, Eleonora Costamagna ${ }^{a}$, Laurence Kenney ${ }^{a}$, Dave Howard ${ }^{\mathrm{a}, \mathrm{b}}$

${ }^{a}$ Centre for Health Sciences Research, School of Health \& Society, University of Salford, Salford, Greater Manchester, UK

${ }^{\mathrm{b}}$ School of Computing, Science and Engineering, University of Salford, Salford, Greater Manchester, UK

c Biomedical Engineering, Delft University of Technology, Netherlands

* Corresponding Author: s.thies@salford.ac.uk

Keywords: older adults, walking aids, design, stability, falls

Word Count: 2998 words, 6 Figures (.tif)

Declaration of Interest: The walking frames were provided by Simply Med Ltd., however, Simply Med Ltd. was NOT involved in study design, data collection \& analysis, interpretation of findings, and writing of this manuscript.

Acknowledgements: This work was supported by The Dowager Countess Eleanor Peel Trust. The Dowager Countess Eleanor Peel Trust had no involvement in the study design, in the collection, analysis and interpretation of data; in the writing of the manuscript; or in the decision to submit the manuscript for publication. We thank Simply Med Ltd. for their provision of the walking frames.

\section{Author Contributions:}

ST: Conceptualization; Data curation; Formal analysis; Funding acquisition; Investigation; Methodology; Project administration; Resources; Supervision; Roles/Writing - original draft.

RR: Data curation; Investigation; Methodology; Resources; Roles/Writing - review \& editing.

AA: Formal analysis; Investigation; Software; Roles/Writing - review \& editing.

TB: Formal analysis; Investigation; Software; Roles/Writing - review \& editing.

BA: Data curation; Investigation; Methodology; Resources; Software; Roles/Writing - review \& editing.

EC: Data curation; Investigation; Methodology; Resources; Software; Roles/Writing - review \& editing.

LPJK: Conceptualization; Funding acquisition; Investigation; Methodology; Supervision;

Roles/Writing - review \& editing.

DH: Conceptualization; Funding acquisition; Investigation; Methodology; Supervision;

Roles/Writing - review \& editing. 


\section{Abstract (294 words)}

\section{Background}

Walking aids are designed for structural support during walking, however, surprisingly self-reported use of a walking aid ("Yes, I use one.") has been identified as a risk factor for falling. Adjustment and design of walking aids may affect their usefulness in facilitating a stable walking pattern. We previously identified that increased body weight transfer onto a walking frame ('device loading') is associated with increased user stability.

\section{Research Question}

We asked: "Could adjustment of walking frame height to a lower height than clinically recommended serve as a mechanism to facilitate device loading and thereby increase stability? And: "Do ultra-narrow frames have an adverse effect on stability as compared to standard-width frames?

\section{Methods}

Ten older adults that were users of front-wheeled walking frames walked with walking frames of 1)'standard width, standard height', 2)'standard width, low height', 3)'narrow width, standard height'. Smart Walker technology was used to record forces acting on the walking frame and inside the user's shoes, and cameras recorded relative position of the user's feet in relation to the frame's feet. Stability of the user-frame system and device loading (percent body weight transferred onto the frame) were calculated. A general linear mixed effects model was used for statistical analysis.

\section{Results}

A lower height setting did not increase device loading and stability, therefore adjusting the height to a lower setting proved to be an unsuccessful mechanism to increase stability. However, device loading was positively correlated with stability for all frame conditions $(p<0.05)$. Finally, stability was reduced when walking with the ultra-narrow, as compared to standard-width, frame $(p=0.002)$. 


\section{Significance}

To increase stability in fall-prone users, active encouragement to transfer body weight onto the walking frame is needed. Considering the adverse effects of ultra-narrow frames on stability, such frames should be prescribed and used with caution. 


\section{Introduction}

Falls in older adults pose a significant global health problem; approximately $40 \%$ of those age 65 and above who live at home experience a fall at least once per year, and in forty falls one leads to hospitalisation [1]. The frequency of falling increases with age as does the severity of the outcomes, moreover, falls are major cause of death in older adults $[1,2]$. It has been estimated that falls cost the UK National Health Service approximately $£ 2.3$ billion per year [3] and the impact of a fall on the individual and their family can be overwhelming [4]. Considering our ageing world population, urgent action is needed to reduce the number of falls in older people.

It has been reported that nearly half the falls experienced by community-dwelling residents happen whilst walking [5]. A walking aid may provide structural support during walking and indeed $22 \%$ of older people use a walking aid indoors and $44 \%$ use one outdoors [6]. However, alarmingly, and rather counterintuitively, self-reported use of a walking aid (i.e. "yes, I use a walking aid") has been identified as a risk factor for falling $[7,8]$. Moreover, others reported fall-related injuries due to falling "whilst using" the device [9].

Although walking aid users are usually those at higher risk of falling, the expectation is that the walking aid reduces that risk. Interestingly, a review article suggested that lifting of the walking aid, its collision with obstacles or the person's feet (e.g. during compensatory stepping in the lateral direction) may contribute to loss of balance [10]. The article highlighted the fact that control of the CoM motion has not been investigated during assisted walking and concluded that "more research is needed to identify and solve specific problems" with walking aids in order to improve "design and guidelines for safer use" [10]. Guidance is often simplistic, for example, to adjust a walking frame the guidance simply states "when standing holding the hand grips, the elbows should be slightly bent. When standing with hands at the side of the body, the handgrips should reach just above the wrist joint." (as stated in the guidance leaflets from the Oxford Health NHS Foundation Trust and also walking aid manufacturer Trulife). However, whether this recommended adjustment indeed facilitates stable use of the walking frame is unknown. Moreover, 
models vary in width. In fact, width is being marketed as a desirable design feature in "slim/ultra-narrow" frame models. However, to date only a few research studies have investigated walking frame design in fallsrisk relevant experimental paradigms [11,12], and no studies exist that investigated adjustment and design of the device in relation to stability during walking.

In order to address these challenging problems, we have developed a method and associated instrumentation ("Smart Walkers") to objectively assess stability of walking frame use [13]. Our approach measures the forces through all feet in contact with the ground (both anatomical feet and feet of the walking frame), together with their location relative to each other to estimate a margin of stability; the lower the margin, the more unstable is the user-walking aid system (see [13]). Using this approach, our recent work [14] has found that placing more body weight onto a walking frame improves user stability across a wide range of tasks such as turning which had previously been reported to be problematic [15]. This insight of increased stability for increased device loading is of relevance to walking frame use, but training users to consciously place more body weight onto the device may prove difficult in this population due to age-related physical and cognitive decline [16]. Alternatively, adjustment of the height of the frame may result in postural change and increased device loading, which may in turn lead to increased stability.

Informed by the above, it was this study's aim to use our Smart Walker technology to investigate, in a group of older adults that are users of walking frames, the effects of walking frame height and width on stability. Hence the project had the following two core objectives:

1. To investigate effects of walking frame height on the stability. It was hypothesized that setting the walking frame to a lower height (one hole, i.e. $2 \mathrm{~cm}$, lower than clinically recommended) would increase stability (i.e. by facilitating body weight transfer and moving the centre of pressure of the person-walking frame system forward, where the system's base of support is generally wider).

2. To investigate effects of walking frame width on stability. It was hypothesized that an ultra-narrow frame, the width of which is $8 \mathrm{~cm}$ less than a standard frame, would reduce stability of the userwalking frame system, as it would tend to reduce the width of the convex polygon enclosing anatomical and frame feet. 
Additionally, we investigated the effects of walking frame height and width on the frequency with which incorrect use of the walking frame occurred: clinical guidance leaflets recommend the user push/glide a front-wheeled frame forward rather than lift it, and our previous work demonstrated that deviation from this guidance negatively impacts on stability [17]. Finally, to solidify findings of our previous work that identified a positive relationship between device loading (\% body weight transferred onto the frame) and stability [14], we once more investigated this relationship within each frame condition.

\section{Methods}

\subsection{Participants}

Ten older adults that were users of front-wheeled walking frames [age (mean \pm SD): 78.9 \pm 8.6 , gender: 6 female \& 4 male, body weight (mean $\pm S D$ ): $79.1 \pm 25.0 \mathrm{Kg}]$ gave written informed consent and participated in the study. All participants were residents in care homes. The sample size was constrained by the challenges associated with recruitment of fall-prone populations and the resources and duration of this project. The study was approved by the University of Salford's Ethics Committee (HSCR16/35, HSCR13/48) and the London Dulwich Research Ethics Committee (16/LO/0986).

\subsection{Experimental Protocol}

Three walking frame conditions for use of a front-wheeled walker were assessed:

1. 'Standard Width, Standard Height', also referred to as 'Baseline': Walking with a front-wheeled walking frame of standard width and depth (width: $57 \mathrm{~cm}$, depth: $54 \mathrm{~cm}$ ) and with its height adjusted as clinically recommended: "When standing holding the hand grips, your elbows should be slightly bent. When standing with your hands at your side the handgrips should reach just above the wrist joint." (Oxford Health NHS Foundation Trust).

2. 'Standard Width, Low height': Walking with a front-wheeled walking frame of standard width same as above, but with its height set to 1 hole below what was clinically recommended, with holes being approximately $2 \mathrm{~cm}$ apart. 
3. 'Narrow Width, Standard Height': Walking with a so-called ultra-narrow model of a front-wheeled walking frame (width: $49 \mathrm{~cm}$, depth: $53 \mathrm{~cm}$ ) but with its height adjusted as clinically recommended. Participants were asked to get up from a chair, then grab the handles of the walking frame and begin walking along a marked path in the gait laboratory that included straight line walking, followed by a left and then a right turn, and then continued with straight line walking before sitting down again (see Figure 1 in [17]; but note that participants in this study omitted the $3^{\text {rd }}$ turn and instead kept walking in a straight line up to a chair). The pathway was modelled in its dimensions after the university's Activities of Daily Living flat: it was representative of walking from the kitchen through the lounge and into the bathroom. Participants performed 1 trial per walking frame condition with a 5-10 minute break between conditions. We note that as these were frail older adults, multiple trials were not feasible.

\subsection{Instrumentation and Data Analysis}

As described above, a walking frame of standard width, and another that was an ultra-narrow frame, were fitted with the required Smart Walker instrumentation (Figure 1). Our Smart Walker approach treats the user and the walking frame as a single combined system for which stability is determined. The Smart Walker System includes four load cells that record forces through the walker's feet, and pressure-sensing insoles inside the user's shoes. The load cells and insole data are synchronized with data from 3D optoelectronic cameras which record the relative position of the user's feet in relation to the feet of the frame. We defined in our previous work $[13,14,17]$ :

- The combined Base of Support (BoS) of the user-frame system: the convex polygon formed by the boundaries of the anatomical and walking frame feet in contact with the ground and the interconnecting lines between them.

- The combined Centre of Pressure (CoP): the point through which the resultant ground reaction force for all feet of both the walking frame and user acts if the resultant moment acts only around an axis perpendicular to the ground plane.

- The combined stability margin 'SM' of the user-frame system: as the distance between the system's CoP and the nearest edge of the BoS. From that, we compute the minimum value of the stability margin ' $\mathrm{SM}_{\min }$ ' 
for each single or dual support period. $\mathrm{SM}_{\min }$ occurs at the instant when the system is closest to "tipping over".

The data further allow for calculation of single and dual support periods of the anatomical feet (i.e. a foot is considered in ground contact when $20 \%$ of the insole sensors are activated), and the system's data also enable identification of the time periods where the frame's wheels are airborne, which is clinically not recommended. Finally, the system allows for calculation of device loading, i.e. the \% body weight transferred onto the frame (indicating the support the person receives from their device), a measure that healthcare professionals had previously perceived as important (see Additional File of reference [17]).

All outcome measures were calculated with custom-written software programmed in Matlab ${ }^{\circledR}$ version R2018a. Outcomes were calculated separately for single and dual support of gait, and averages for each outcome measure and each gait phase were obtained.

\subsection{Statistical Analyses}

All $\mathrm{SM}_{\min }$ data passed checks for normality with ratios of skewness/standard error of skewness and kurtosis/standard error of kurtosis lying within $+/-2$. To assess effects of the frame condition and effects of correct/incorrect use on stability, a general linear mixed effects model was used for statistical analysis. For this the $\mathrm{SM}_{\min }$ data obtained for single and dual support were analysed in SPSS; 'Frame Condition' (standard frame, low frame, narrow frame) and 'Correctness' (i.e. correct versus incorrect use) were modelled as fixed effects. Moreover, the 'Person ID' was included as a random effect because participants may differ in their physical abilities (and hence their stability), for example, due to age or disabilities/co-morbidities.

Furthermore, to assess whether the frame condition had an effect on the device loading, and also to assess effects of the frame condition on the frequency of incorrect use occurring, repeated measures ANOVAs were used, and in the specific case of device loading during single support a Friedman test was used due to deviations from normality for this particular data set. Finally, relationships between device loading and 
$\mathrm{SM}_{\min }$ were investigated within frame condition using Pearson's Correlations, and for the specific case discussed above a Spearman Rank's Correlation was used due to deviation of the data's distribution from normality. All statistical analyses were done in IMB SPSS Statistics 25.

\section{Results}

\subsection{Stability and device loading}

The stability margin $\mathrm{SM}_{\min }$ was reduced for the low frame by $4.8 \%$ and for the narrow frame by $20.9 \%$ as compared to baseline 'standard height, standard width' during the single support of gait, and during dual support $\mathrm{SM}_{\min }$ was reduced by $1.5 \%$ and $14.9 \%$, compared to baseline, for the low and the narrow frame respectively (Figure 2). Comparing the narrow frame to baseline and to the low frame resulted in $p$ values of 0.002 and 0.024 , respectively. We also note that one participant was not able to walk with the narrow frame, stating "I feel like I am falling over".

Other differences between means were small and had $p$ values greater than 0.05 , including comparison of $S M_{\min }$ at baseline to $S M_{\min }$ for the low frame during single support, and all $S M_{\min }$ comparisons for dual support.

Device loading (\% body weight transferred onto the frame) was reduced for the lower and the narrow frame as compared to baseline for both, the single and the dual support phases of gait (Figures 3), however, comparisons were associated with $p$ values greater than 0.05 . Notably, a positive relationship between stability and body weight transfer was observed for all frame conditions ( $p<0.05$ for four frame conditions, see Figure 4).

\subsection{Incorrect use and stability}

The $\%$ of single and dual support phases where incorrect use was the case showed large variability in the data (Figure 5) and did not result in significant differences for comparison of the three frame conditions, with all $p$ values greater than 0.05 . Notably, the bars in Figure 6 show that $S M_{\min }$ values were generally lower for incorrect use as compared to correct use for all frame conditions in single and dual support; 
however, a $p$ value of less than 0.05 was only obtained for single support $(p=0.004)$ whilst in dual support the associated $\mathrm{p}$ value was 0.065 .

\section{Discussion \& Conclusions}

Design of walking aids in relation to falls-risk has received little attention to date. Two studies previously reported on design features of a walking frame in relation to balance recovery [11,12], leading to the conclusion that specific design aspects increase the risk of collision of the lower limbs with the aid [12]. This project adds to the existing knowledge and is the first to report on the safety implications of recommended height adjustment and width of walking frames.

In disagreement with our first hypothesis, we found that our stability measure $\mathrm{SM}_{\min }$ was lower when the frame was set to a lower height than standard, and this was observed during both, single and dual support. One underlying reason why we did not achieve increased stability for the lower frame is that the body weight transferred onto the frame was not increased as we had initially expected. This indicates that simply adjusting the frame to a lower height setting does not result in the user putting more weight onto the frame, and future work should investigate whether this may have been due to changes in trunk or hip angle, and whether the lack of device loading may reflect a strategy to ensure friction force is low enough to allow the frame to slide over the ground. Nevertheless, in line with our previous findings [14], positive relationships between $\mathrm{SM}_{\min }$ and device loading were obtained for all walking frame conditions, confirming that increased body-weight transfer onto the frame is associated with increased $\mathrm{SM}_{\min }$ values. Since $\mathrm{SM}_{\min }$ reflects how close the user-device system is to tipping over, a strategy of encouraging the user to consciously place more weight onto the frame may be explored where increasing stability is of interest (for example, during performance of challenging tasks such as opening doors or walking backwards to back up towards a chair, or for those users that have a high falls-rate despite using their device). However, users would need to be cognitively able to follow instructions, and we also acknowledge that increased body 
weight transfer onto the frame may have an adverse effect on lower limb muscle strength, hence the benefit of increasing $\mathrm{SM}_{\min }$ has a trade-off which must be carefully considered.

In agreement with our second hypothesis, stability was reduced when walking with the narrow, as compared to the standard-width, frame. There was also a tendency for participants to transfer less body weight onto the narrow frame as compared to the standard-width frame. It is further noteworthy that one participant did not feel safe/stable enough to walk with the narrow frame. Considering these findings, the narrow frame presents a trade-off: it may be easier to use in constrained spaces; however, it reduces stability as compared to a standard-width frame.

Incorrect use, defined as the front wheels of the frame being lifted during walking, differed only a little between frame conditions; we also note that variability within that data, shown by the error bars in Figure 5, reflected high inter-subject variations regarding incorrect use. Notably, incorrect use was associated with decreased stability during single support in particular ( $p$ less than 0.05 ). Hence lifting of the front wheels is to be avoided especially during single support. This is in agreement with our earlier findings [17] which also highlighted that reasons for lifting are at least in part driven by design flaws of the front-wheeled walker: its front wheels are fixed and cannot swivel, and this appears to encourage lifting of the walker when turning.

We acknowledge that the small sample size $(n=10)$ and use of only one type of walking frame (i.e. a frontwheeled walking frame) limit the generalizability of findings to the larger population of walking aid users. Future work should explore how to facilitate participation of this vulnerable population in biomechanical studies, and effects of design features, including hand grips and wheel design, merit investigation in relation to both, stability and device loading. Furthermore, whilst reporting the combined stability margin of user and device has demonstrated advantages [13], we acknowledge that our approach prevents us from defining a clear direction of the system's stability margin because at times the frame and the user may be at an angle to one another (i.e. when the frame is rotated before the user's feet change direction). Hence, 
whilst the system's SM is mechanically insightful it cannot inform on stability in a particular plane, as a plane for the system cannot be consistently defined in trials that include turning.

In conclusion, simply adjusting the frame height to a lower setting did not increase stability through increased body weight transfer. However, the percentage of body weight placed on the frame was positively correlated with stability, hence actively encouraging this behaviour may help those users that are prone to falling. Narrow frames are to be prescribed and used with caution as they decrease stability as compared to walking with a standard-width frame. Finally, lifting of the frame should be discouraged as it reduces stability, and the industry should consider redesigning the front-wheeled walker to facilitate turning without lifting.

\section{Conflict of Interest Statement}

The authors have no financial and personal relationships with other people or organisations that could inappropriately influence (bias) their work. 


\section{References}

[1] L.Z. Rubenstein, Falls in older people: epidemiology, risk factors and strategies for prevention, Age Ageing 35 Suppl 2 (2006) ii37-ii41. https://doi.org/10.1093/ageing/afl084

[2] NHS. Falls. 2014 cited 2014 May: http://www.nhs.uk/conditions/Falls/Pages/Introduction.aspx (Accessed 8 November 2019).

[3] NICE, Falls: assessment and prevention of falls in older people, 2013. Available from: https://www.nice.org.uk/guidance/cg161 (Accessed 8 November 2019).

[4] M.C. Faes, M.F. Reelick, L.W. Joosten-Weyn Banningh, Md. Gier, R.A. Esselink, M.G. Olde Rikkert, Qualitative study on the impact of falling in frail older persons and family caregivers: foundations for an intervention to prevent falls, Aging Ment. Health 14 (2010) 834-842.

https://doi.org/10.1080/13607861003781825.

[5] W.R. Berg, H.M., Alessio, E.M. Mills, C. Tong, Circumstances and consequences of falls in independent community dwelling older adults, Age Ageing 26 (1997) 261-268. https://doi.org/10.1093/ageing/26.4.261.

[6] C. Löfqvist, C. Nygren, A. Brandt, F. Oswald, S. Iwarsson, Use of mobility devices and changes over 12 months among very old people in five European countries, Aging Clin. Exp. Res. 19 (2007) 497-505.

[7] S. Deandrea, E. Lucenteforte, F. Bravi, R. Foschi, C. La Vecchia, E. Negri, Risk factors for falls in community-dwelling older people: a systematic review and meta-analysis, Epidemiology 21 (2010) 658-668.

[8] B.A. West, G. Bhat, J. Stevens, G. Bergen, Assistive device use and mobility-related factors among adults aged $\geq 65$ years, J. Safety Res. 55 (2015) 147-150. https://doi.org/ 10.1016/j.jsr.2015.08.010.

[9] B.J. Hefflin, T.P. Gross, T.J. Schroeder, Estimates of medical device-associated adverse events from emergency departments, Am. J. Prev. Med. 27 (2004) 246-253.

https://doi.org/10.1016/j.amepre.2004.04.005.

[10] H. Bateni, B.E. Maki, Assistive devices for balance and mobility: benefits, demands, and adverse consequences, Arch. Phys. Med. Rehabil. 86 (2005) 134-45. https://doi.org/10.1016/j.apmr.2004.04.023.

[11] H. Bateni H, E. Heung, J. Zettel, W.E. McLlroy, B.E. Maki, Can use of walkers or canes impede lateral compensatory stepping movements?, Gait Posture 20 (2004) 74-83. https://doi.org/ 10.1016/S09666362(03)00098-5.

[12] B.E. Maki, K.C. Cheng, A. Mansfield, C.Y. Scovil, S.D. Perry, A.L. Peters, S. McKay, T. Lee, A. Marquis, P. Corbeil, G.R. Fernie, B. Liu, W.E. Mcllroy, Preventing falls in older adults: new interventions to promote more effective change-in-support balance reactions, J. Electromyogr. Kinesiol. 18 (2008) 243-254. https://doi.org/10.1016/j.jelekin.2007.06.005.

[13] E. Costamagna, S.B. Thies, L.P.J. Kenney, D. Howard, A. Liu, D.A. Ogden, A generalisable methodology for stability assessment of walking aid users, Med. Eng. Phys. 47 (2017) 167-175.

https://doi.org/10.1016/j.medengphy.2017.06.013.

[14] E. Costamagna, S.B. Thies, L.P.J. Kenney, D. Howard, U. Lindemann, J. Klenk, R. Baker, 2018. Objective measures of rollator user stability and device loading during different walking scenarios, PLoS One. 14, e0210960. https://doi.org/10.1371/journal.pone.0210960.

[15] U. Lindemann, M. Schwenk, J. Klenk, M. Kessler, M. Weyrich, F. Kurz, C. Becker, Problems of older persons using a wheeled walker, Aging Clin. Exp. Res. 28 (2016) 215-220. https://doi.org/ 10.1007/s40520015-0410-8. 
[16] J.A. Chase. Methodological challenges in physical activity research with older adults. West. J. Nurs. Res. 35 (2013) 76-97. https://doi.org/ 10.1177/0193945911416829

[17] S.B. Thies, A. Bates, E. Costamagna, L.P.J. Kenney, M. Granat, J. Webb, D. Howard, R. Baker, H. Dawes, Are older people putting themselves at risk when using their walking frames?, BMC Geriatrics, under review. 


\section{Figure Captions}

Figure 1. Smart Walker instrumentation set up. The forces through each of the frame's feet are measured by load cells, and the forces through the participant's feet are estimated using data from a pressure-sensing insole in each shoe. The relative location of the frame's and participant's feet are derived from tracking reflective markers on the participant's shoes and the frame. These data are used to calculate the combined base of support, combined centre of pressure, combined stability margin and periods of contact for the frame's and participant's feet. For further details we refer readers to [13].

Figure 2. Average minimum stability margin $\mathrm{SM}_{\min }$ during single (left) and dual (right) support periods for the three frame conditions.

Figure 3. Device loading (\% body weight transferred onto the frame) during single (left) and dual (right) support periods for the three frame conditions.

Figure 4. Correlation between device loading (\% body weight transferred onto the frame) and minimum stability margin $\mathrm{SM}_{\min }$ during single support (left) and dual support (right) for all three frame conditions (top to bottom).

Figure 5. The \% of single support (black bars) and dual support (grey bars) phases where incorrect use was the case, shown for all three frame conditions.

Figure 6. Stability margin $\mathrm{SM}_{\min }$ for correct versus incorrect use and during both single support and dual support for all three frame conditions. 


\section{Figures}

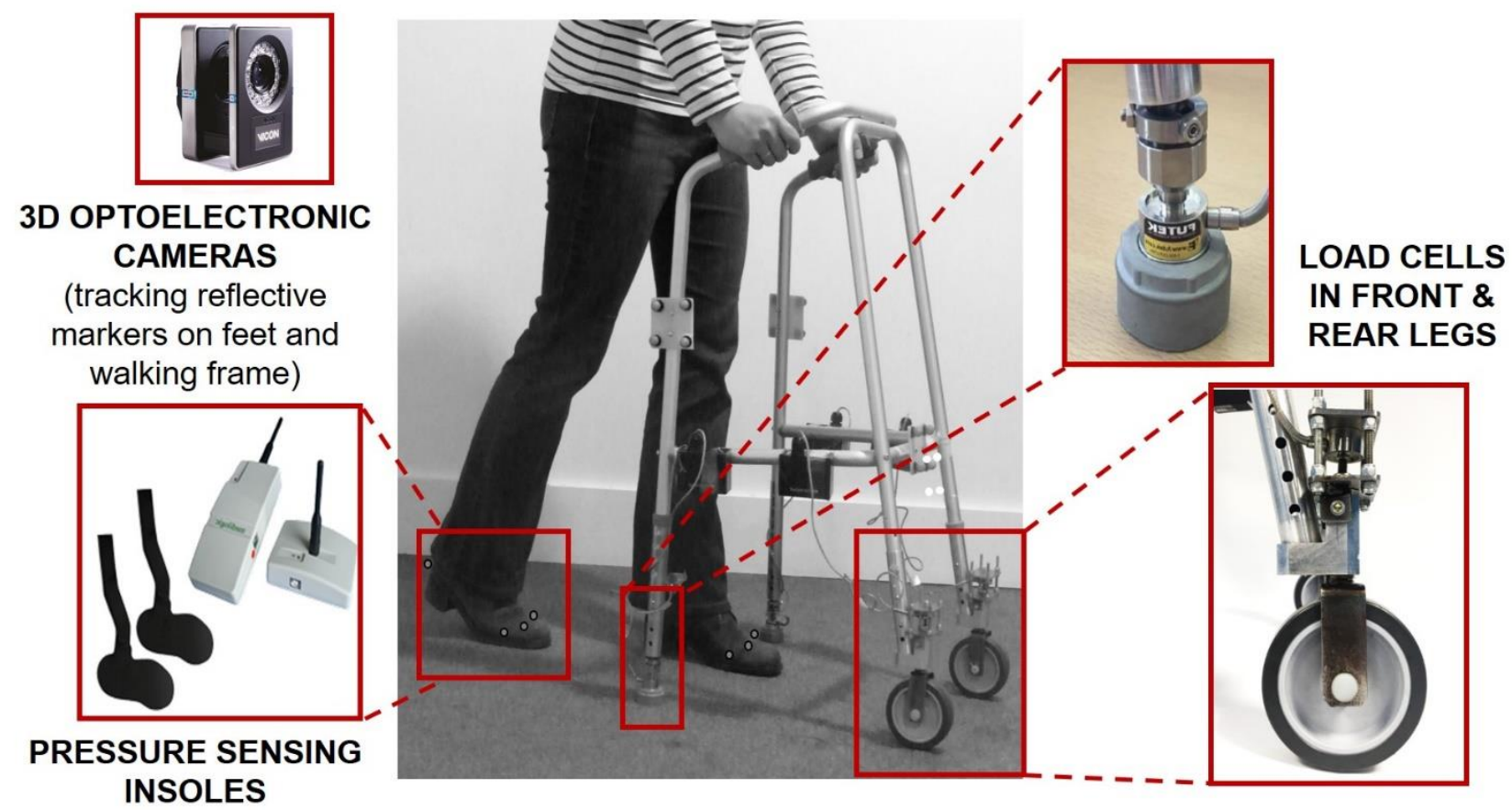

Figure 1.
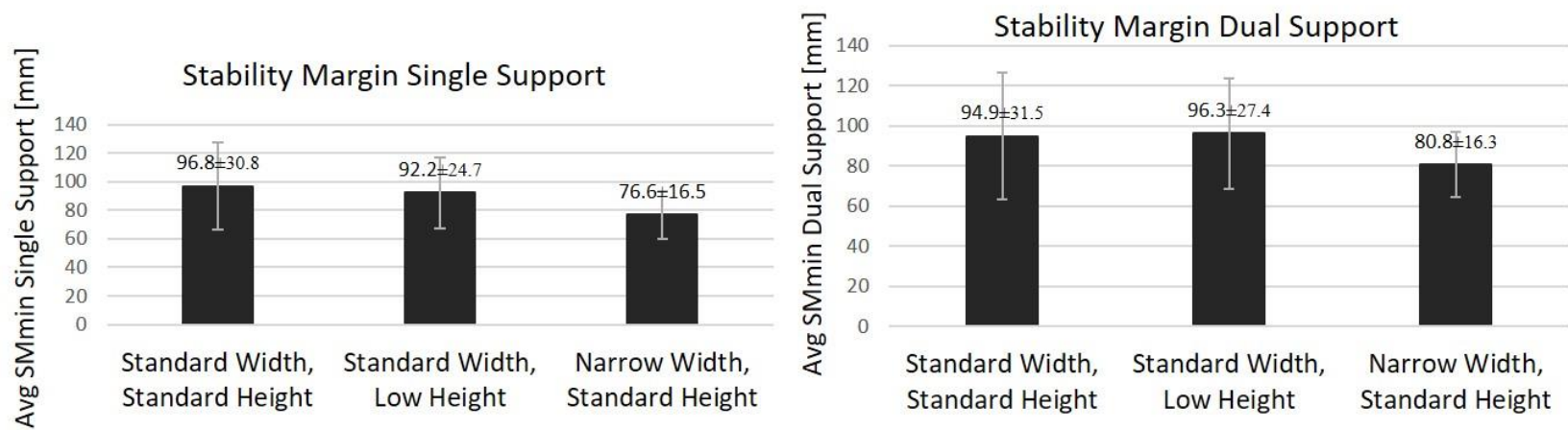

Figure 2.
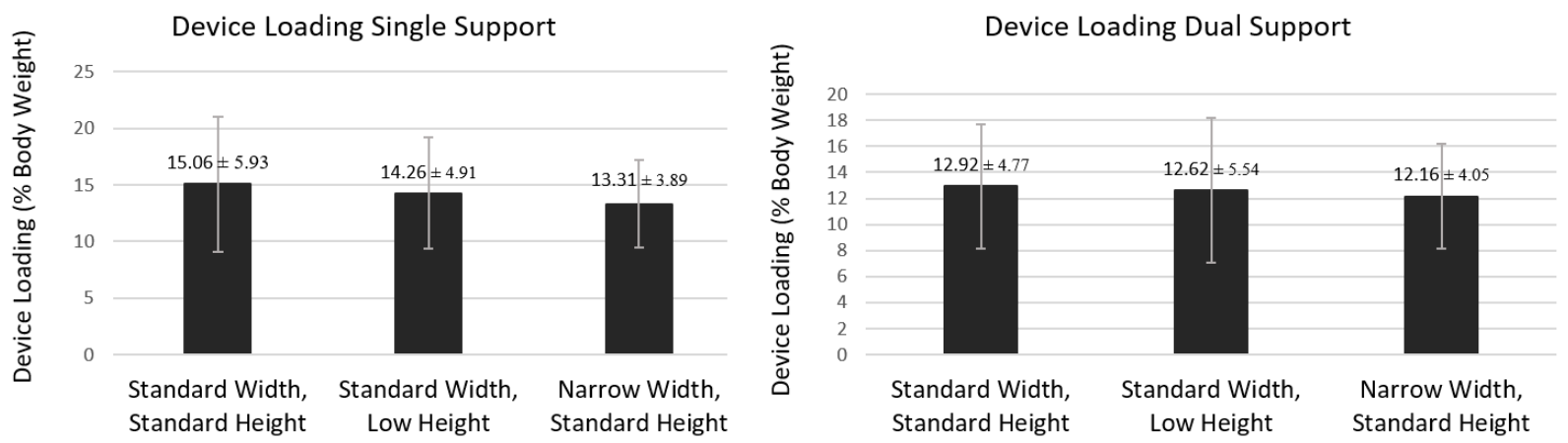

Figure 3. 
Single Support
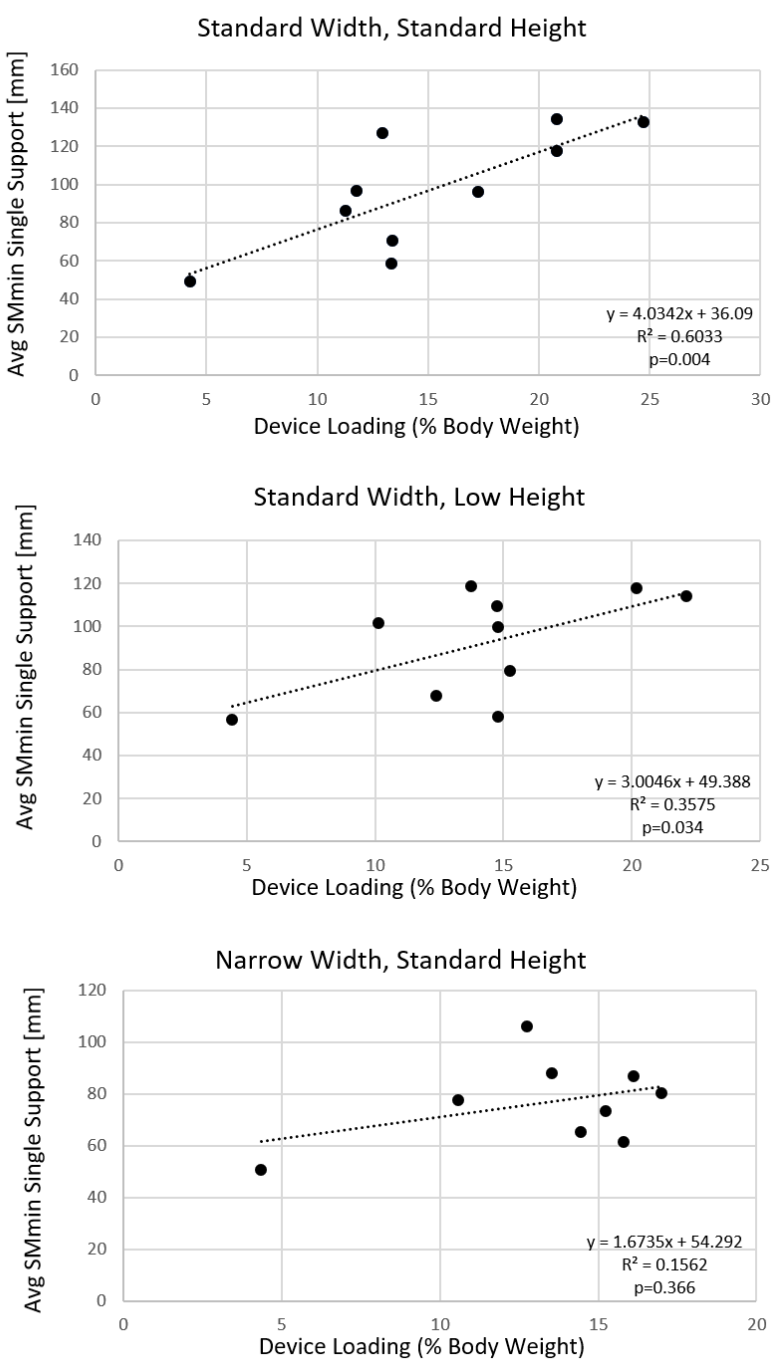

Dual Support

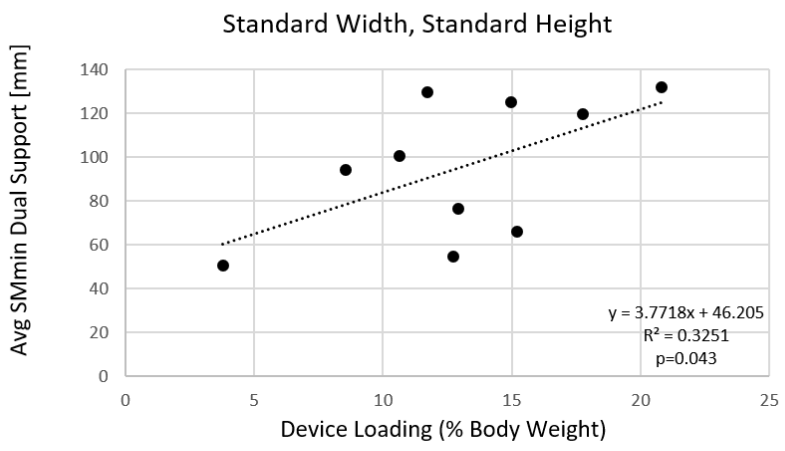

Standard Width, Low Height

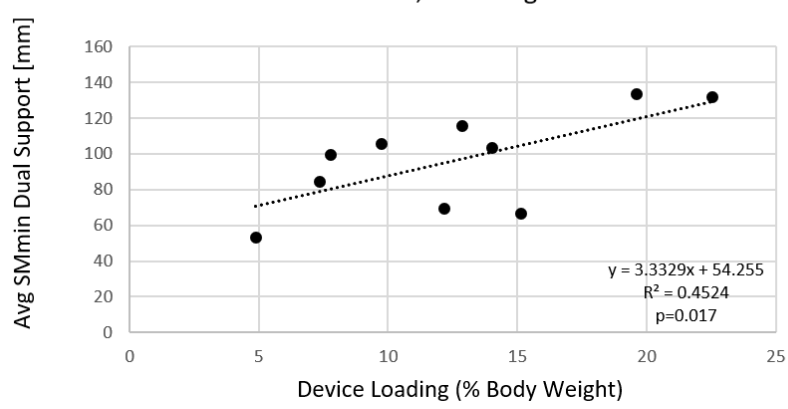

Narrow Width, Standard Height

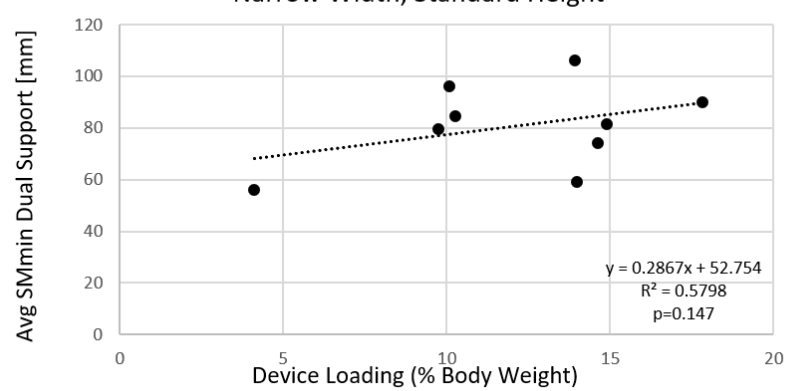

Figure 4. 


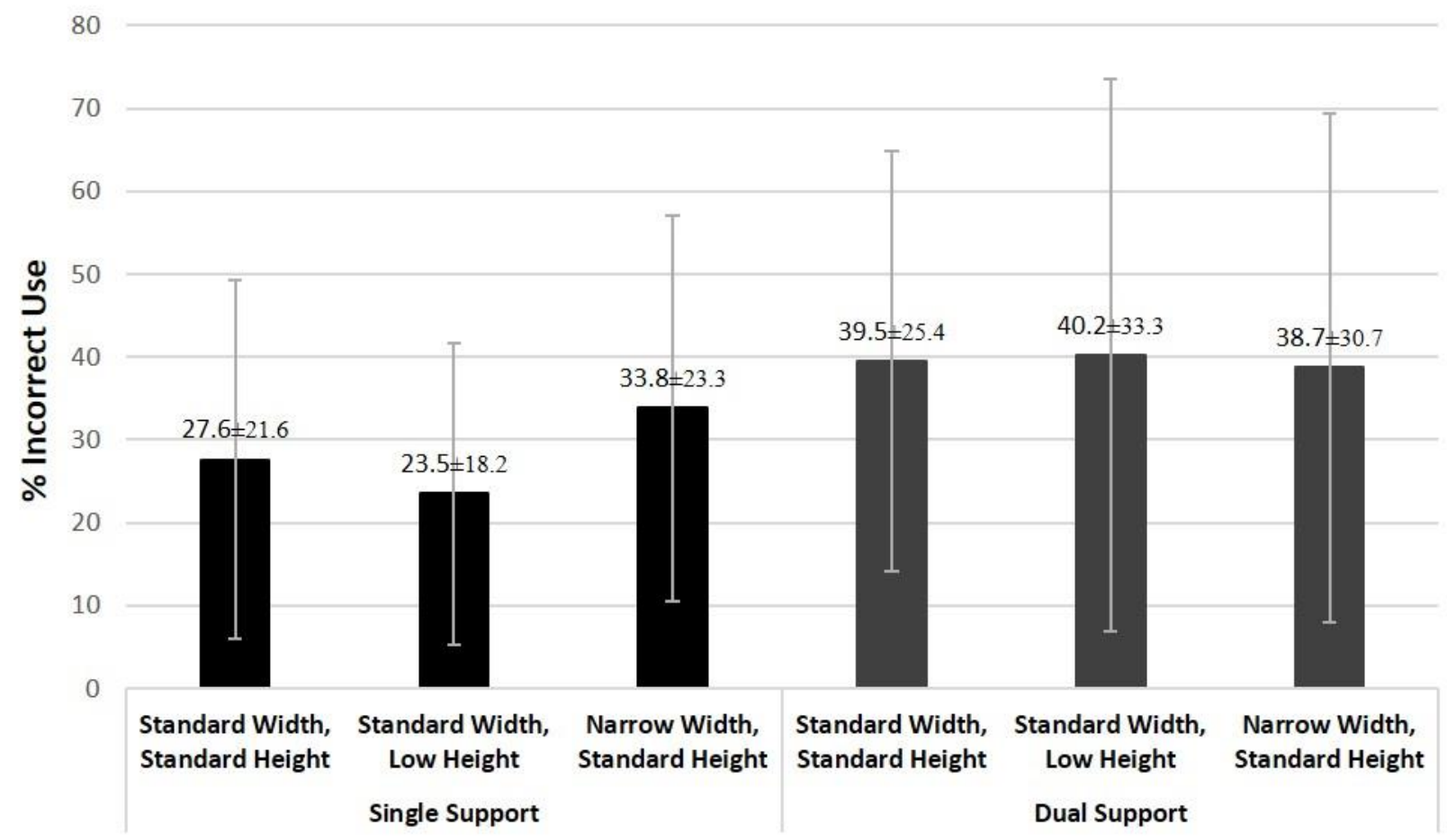

Figure 5.

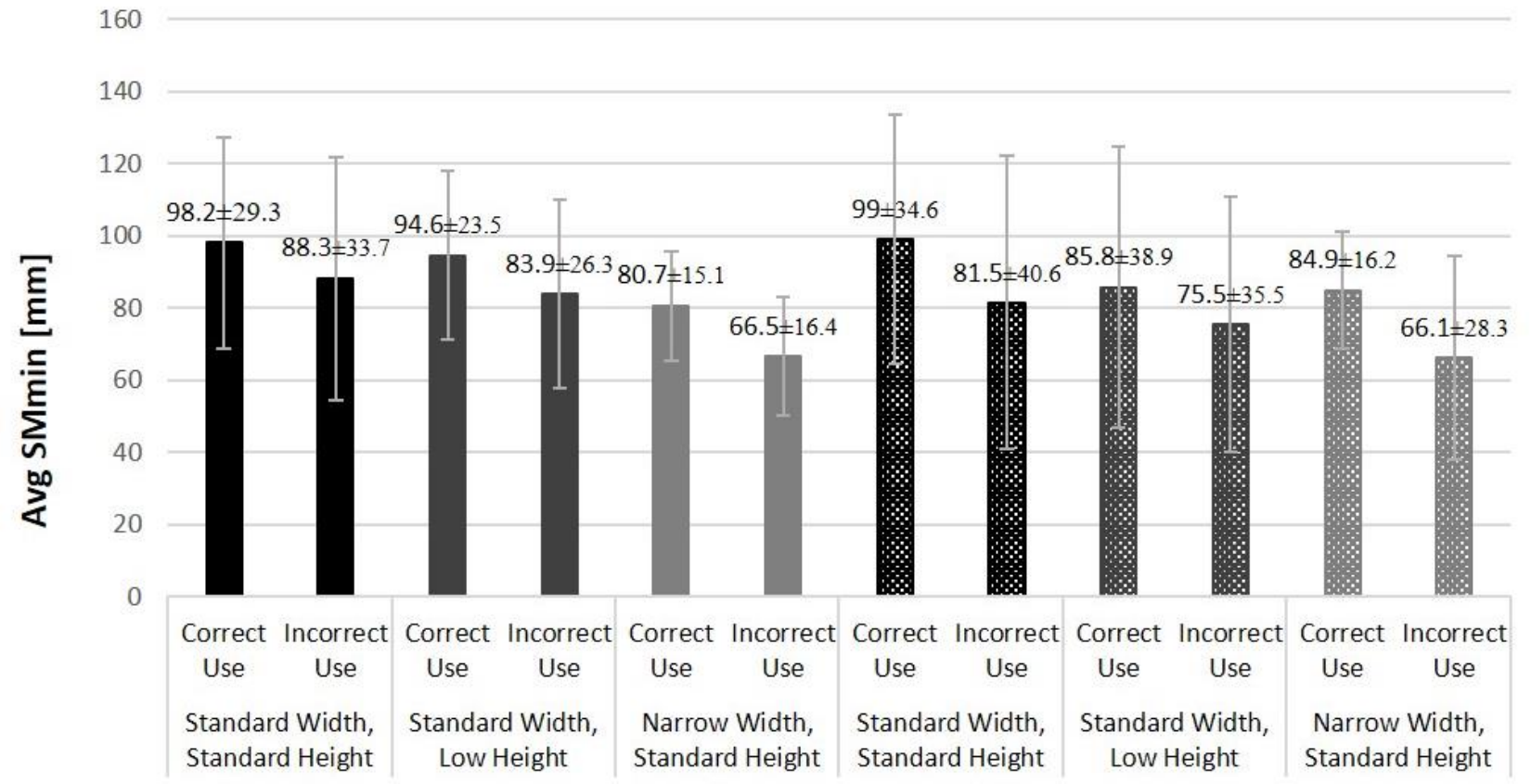

Single Support

Dual Support

Figure 6. 


\section{Highlights}

- A lower height setting did not increase body weight transfer to the walking frame.

- Yet body weight transfer positively correlates with stability.

- Conscious increase in body weight transfer may be suggested to fall-prone users.

- Narrow frames decrease stability and should be prescribed and used with caution. 